\title{
ROYAUME DE MACÉDOINE ET COLONIES GRECQUES : LANGUES ET INSTITUTIONS
}

Chez Homère, ce que nous appelons aujourd'hui la Grèce du Nord était le pays soit des dieux soit des barbares, en tout cas pas celui des Achéens, les futurs Hellènes ${ }^{1}$. Moins de mille ans plus tard, ce même pays, appelé désormais la Macédoine, faisait partie de la Grèce, était déjà devenu en quelque sorte la Grèce

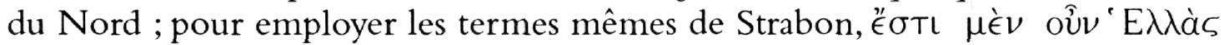

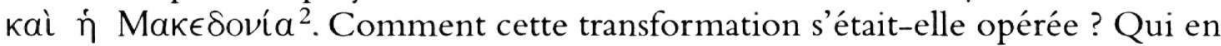
avaient été les auteurs ? C'est Strabon lui-même qui répond à ces questions dans un raccourci saisissant de la préhistoire de cette région : "La Macédoine actuelle était appelée jadis Émathie. Elle doit son nom à un de ses anciens chefs, un certain Makédon... Les peuples qui se partageaient son territoire étaient des Épirotes, des Illyriens, et surtout des Bottiéens et des Thraces. Les Bottiéens, à ce qu'on dit, étaient venus de Crète sous la conduite de Botton. Parmi les Thraces on distinguait les Pières qui habitaient la Piérie et la région de l'Olympe, les Paioniens établis sur les rives du fleuve Axios dans une région qui prend pour cette raison le nom d'Amphaxitis, les Édones et les Bisaltes installés sur le reste du pays jusqu'au Strymon... Tous ces peuples passèrent sous la domination des Argéades et des Chalcidiens d'Eubée ${ }^{3}$.

A. Toynbee, sans avoir relevé ce passage de Strabon, a esquissé dans son œuvre monumentale, $A$ Study of History, les vies parallèles du royaume de Macédoine et de la Ligue Chalcidienne et s'est livré à un fascinant exercice d'histoire-fiction sur ce qu'eût été l'histoire de la Grèce, si les Spartiates n'étaient pas accourus au secours d'Amyntas III en 382 av. J.-C. et n'avaient pas obtenu la dissolution de la Ligue Chalcidienne en $379^{4}$ : " comme le monde hellénique s'agrandissait et que le pouvoir commençait à passer des petits états du centre aux nouveaux états de plus grand calibre sur la périphérie ", écrit-il, «il devint évident qu'une des nouvelles grandes puissances helléniques émergerait dans le voisinage du Golfe Thermaïque (Golfe de Salonique), et de la basse vallée du fleuve Axios (Vardar). La question était de savoir si le noyau de cette nouvelle puissance serait le royaume macédonien, qui avait été fondé sur la plaine côtière à l'Ouest du fleuve par des conquérants grecs macédoniens, qui étaient descendus des hauts plateaux de

${ }^{1}$ Cf. Hom., Il., XIV, 226, avec le commentaire de R. Janko, dans The Iliad : a Commentary, Cambridge, 1992, p.186-87, et Hom., Il., II, 848-50, avec le commentaire de G.S. Kirk, dans The Iliad : a Commentary, Cambridge, 1985, p. 258.

2 Strab., VII, frg. 9.

${ }^{3}$ Strab., VII, frg. 11.

${ }^{4}$ A. Toynbee, A Study of History, vol. III, Londres, New York, Toronto, 1935², p. 477-85. 
l'arrière-pays, ou bien si la nouvelle puissance surgirait des poleis chalcidiennes, qui avaient été fondées sur la péninsule à l'Est du golfe par des colons grecs d'Eubée, qui étaient arrivés d'outre mer comme missionnaires de la nouvelle foi de la polis dans les marches lointaines du monde hellénique " ... "Si ç'avait été le koinon chalcidien et non pas la couronne de Macédoine qui s'était érigé en grande puissance en Grèce du Nord, au IVe siècle av.J.-C. ", poursuit-il, «le cours de l'histoire grecque pendant les deux siècles à venir eût pu être très différent ; car, si une fédération de poleis avait réussi à souder ensemble tous les territoires et tous les peuples réunis sous le sceptre macédonien par le roi Philippe II, cette puissance eût possédé une combinaison de qualités jamais possédées ni par la Macédoine, ni par Thèbes, ni par Sparte, ni par Athènes. Une fédération chalcidienne aurait joui des avantages matériels d'une échelle de grandeur supérieure à la polis - ce dont bénéficiait la Macédoine, mais qui faisait défaut aux trois poleis qui avaient essayé avant elle de réaliser l'union politique de la Grèce, et avaient échoué. En même temps une fédération chalcidienne aurait possédé un avantage spirituel qui manquait à la Macédoine. En tant que fédération de poleis, elle aurait été reconnue comme un état de la même espèce que les poleis historiques du Sud et, par conséquent, Thèbes, Athènes et Sparte, quand elles se seraient pliées à la force infiniment supérieure de la nouvelle Grande Puissance, ne se seraient pas ni aliénées, ni senties humiliées intérieurement comme elles le furent en 338 av. J.-C., par le sentiment qu'elles se soumettaient par force majeure à un régime barbare et réactionnaire qui était la négation même de tout ce que la polis avait représenté depuis son apparition ${ }^{6} "$. Et l'historien britannique de spéculer que si la Ligue Chalcidienne avait réussi à annexer la Macédoine au lieu que ce fût le contraire, une Grèce unie alliée à Hannibal aurait infligé à Rome le sort que celle-ci réserva à la Macédoine en 168 et à Carthage en 146 av. J.-C. ${ }^{7}$

Cet essai d'A. Toynbee, aussi pénétrant et instructif qu'il soit, souffre non seulement du moralisme habituel de l'auteur, mais aussi de l'ignorance, tout à fait naturelle à l'époque où il écrivait, des conséquences qu'avait eues pour la Macédoine la conquête des colonies grecques des côtes septentrionales de la Mer Égée. Mais reprenons les choses dans l'ordre chronologique.

Quoique la présence grecque sur le littoral septentrional de la Mer Égée et tout particulièrement en Chalcidique puisse remonter à la fin de l'époque mycénienne ${ }^{8}$, la fondation des principales colonies dans la région doit se situer vers la fin du VIIIe et au cours du VIIe siècle ${ }^{9}$. C'est précisément l'époque - si l'on se fie aux légendes dynastiques - de la fondation du royaume argéade d'Aigéai par un prince téménide venu d'Argos ${ }^{10}$. Il serait difficile d'imaginer des versions de culture grecque aussi différentes que celles que représentaient ces deux foyers de l'hellénisme de la Grèce du Nord appelés à de si hautes destinées. D'un côté

${ }^{5}$ A. Toynbee, op. cit., p. 479.

${ }^{6}$ A. Toynbee, op. cit., p. 488-89.

${ }^{7}$ A. Toynbee, op. cit., p. 489.

${ }^{8}$ Communications de J.Vokotopoulou encore inédites aux conférences sur Tò ápxaı

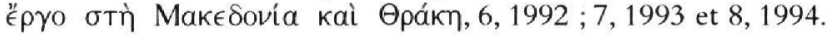

${ }^{9}$ Cf. N.G.L. Hammond, A History of Macedonia, vol. I, Oxford, 1972, p. 424-25 et 440-41.

${ }^{10}$ Cf. N.G.L. Hammond, op. cit., p. 430-35. 
- avec de très rares exceptions (Skionè, Potidée) - des Ioniens insulaires, porteurs des valeurs de la polis déjà éclose et d'une civilisation matérielle et spirituelle complexe et raffinée. De l'autre, un peuple (ethnos) de montagnards parlant un dialecte du type nord-occidental, sur lequel nous allons revenir, à peine sorti du stade transhumant de son développement et encore gouverné par des rois.

Ici je dois ouvrir une parenthèse. Pendant longtemps on s'était fait des idées fausses sur l'origine aussi bien de l'un que de l'autre protagoniste de l'histoire de

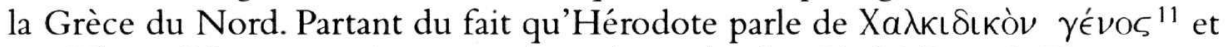
que Thucydide ne mentionne pas les métropoles des Chalcidiens de Thrace, une série de savants, dont le représentant le plus récent fut M. Zahrnt, dans son excellent ouvrage Olynth und die Chalkidier, avait soutenu que ces Chalcidiens de Thrace n'étaient pas venus d'Eubée, mais représentaient une branche des Ioniens, qui lors de leur migration au second millénaire av. J.-C. ne serait pas descendue jusqu'au Sud de la péninsule hellénique, mais se serait fixée en Chalcidique. Cette théorie trouverait appui dans le dialecte et le calendrier de cette région, tels qu'ils nous sont révélés par les inscriptions ${ }^{12}$. L' accroissement du matériel épigraphique et son examen systématique pendant ces dernières années ont permis à $\mathrm{D}$. Knoepfler ${ }^{13}$ et à moi-même ${ }^{14}$, travaillant de façon indépendante, d'apporter la preuve qu'il n'en était rien et que la langue et les institutions des Chalcidiens de Thrace avaient bel et bien leur origine en Eubée.

D'autre part, un courant historiographique beaucoup plus ancien et puissant contestait l'origine grecque des fondateurs du royaume macédonien ${ }^{15}$. À ce débat scientifique tout à fait justifié, vu l'indigence de nos sources, au moins jusqu'à une époque récente, est venue se greffer une querelle politique et diplomatique affectant les écrits historiques non seulement chez les protagonistes locaux des conflits balkaniques, mais aussi, dans beaucoup de pays du monde entier dont des ressortissants, à un moment ou un autre, d'une façon ou d'une autre, se sont trouvés impliqués dans les rivalités des peuples de la région ${ }^{16}$. Le débat portait à la fois sur la langue, la religion, les institutions politiques et les mœurs des Macédoniens. Deux développements des dernières décennies met-

${ }^{11}$ Hérod., 7, 18, 2 et $8,127$.

${ }^{12}$ Voir M. Zahrnt, Olynth und die Chalkidier, dans Vestigia, 14, Munich 1971, p. $12-27$ et M.B. Hatzopoulos, Actes de vente de la Chalcidique centrale (MEAETHMATA, 6), Athènes 1988, p. 40-70, avec bibliographie.

13 D. Knoepfler, Le calendrier des Chalcidiens de Thrace, essai de mise au point sur la liste et l'ordre des mois eubéens, dans Journal des Savants, 1989, p. 23-59 ; id., The calendar of Olynthus and the Origin of the Chalcidians in Thrace, dans Greek Colonists and Native Populations, Canberra-Oxford 1990, p. 99115.

${ }^{14}$ M.B. Hatzopoulos, op. cit., p. 40-70.

15 Pour un survol commode de l'immense bibliographie, voir J.N. Kalléris, Les anciens Macédoniens, Athènes, 1954, p. 11-47 et M.B. Sakellariou, The Nationality of the Macedonians, dans Macedonia : 4000 Thousand Years of Greek History and Civilization, Athènes, 1983, p. 48 et 534-35.

${ }^{16}$ Cf. J.N. Kalléris, op. cit., p. 36-44 et E.N. Borza, In the Shadow of Olympus, Princeton, N.J., $1992^{2}$, p. 8-12 et 90-95. Il est intéressant de noter que les auteurs de ces ouvres constituent en même temps de bons exemples de savants impliqués à titres divers de par leurs origines dans les antagonismes locaux. Pour un autre exemple, cf. E. Badian, Greeks and Macedonians, dans Macedonia and Greece in Late Classical and Early Helenistic Times (Studies in the History of Art, vol. 10), Washington, 1982, p. 33-51. 
tent fin - s'ils n'ont déjà fait - à cette controverse, au moins dans sa partie scientifique, sinon politique : d'une part, la véritable explosion de l'archéologie macédonienne, qui a augmenté de manière prodigieuse nos connaissances sur tous les aspects de la Macédoine antique ${ }^{17}$, et, d'autre part, la modification progressive d'une vision trop athénocentrique de l'histoire et de la civilisation helléniques, qui tendait à réduire la Grèce aux limites que lui assignait jadis le grand historien athénien Thucydide, abandonnant au monde barbare tout ce qui sur le continent grec se trouvait au Nord et à l'Ouest de Delphes ${ }^{18}$. Aujourd'hui nous savons que les fondateurs du royaume macédonien parlaient un dialecte grec, dont la forme écrite était proche de celle des dialectes du Nord-Ouest ${ }^{19}$; qu'ils adoraient - ce qui ne saurait étonner - les dieux de l'Olympe ${ }^{20}$; qu'ils n'ignoraient pas les institutions civiques, voire que leur royauté ne différait guère des royautés des peuples voisins, Epirotes ou Thessaliens ${ }^{21}$; enfin, que leurs mœurs sociales n'étaient pas étrangères aux normes panhelléniques, jusques et y compris dans un domaine aussi typique que les classes d'âge des jeunes gens et des jeunes filles ${ }^{22}$.

Nous ignorons presque tout des rapports entre le royaume téménide et les colonies des Grecs du Sud jusqu'au règne d'Amyntas Ier dans la seconde moitié du VIe siècle ${ }^{23}$. Étant donné que le domaine royal était alors confiné à la Bottie et à la Piérie ${ }^{24}$, la question se pose uniquement au sujet des rapports des Macédoniens avec la colonie érétrienne de Méthone. La découverte récente de son site exact tendrait, cependant, à indiquer l'existence de relations amicales ou du moins d'un modus vivendi. En effet, comment expliquer le développement du centre urbain de la colonie à une distance de plus d'un kilomètre de son port,

17 Cf. E.N. Borza, The History and Archaeology of Macedonia : Retrospect and Prospect, dans Macedonia and Greece in Late Classical and Early Helenistic Times (Studies in the History of Art, vol. 10), Washington, 1982, p. 17-30 ; id., op. cit. à la n. 16, p. 12-18 ; M.B. Hatzopoulos, $A$ Century and a Lustrum of Macedonian Studies, dans AncWorld, 4, 1981, p. 91-108; id., Greek and International Scholarship on Ancient Macedonia, some Recent Developments, dans Macedonian Hellenism, Melbourne, 1990, p. 109-115, et surtout les actes des colloques internationaux sur la Macédoine antique organisés tous les cinq ans à Thessalonique : Ancient Macedonia, I-V, Thessalonique, 1970-1993, et les

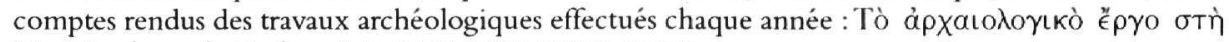

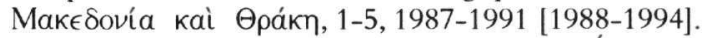

${ }^{18}$ Cf. P. Cabanes, Le pouvoir local au sein des États fédéraux : Épire, Acarnanie, Étolie, dans La Béotie antique, Paris, 1985, p. 343-44.

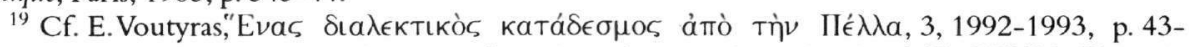
48 ; Bull. Epigr., 1994 ; M.B. Hatzopoulos, Actes de vente d'Amphipolis (MEAETHMATA, 14), Athènes, 1991, p. 79, n. 4.

${ }^{20}$ Pour une synthèse récente sur la vie religieuse en Macédoine, voir M.B. Hatzopoulos et Lilly Kahil, Cultes et Mythes, dans La Macédoine de Philippe II à la conquête romaine, Paris, 1993, p. 106116.

${ }^{21}$ En attendant la parution prochaine de M.B. Hatzopoulos, Macedonian Institutions under the Kings : a Historical and Epigraphic Study, voir du même auteur, Les institutions politiques, dans La Macédoine de Philippe II à la conquête romaine, Paris, 1993, p. 55-57.

22 Cf. M.B. Hatzopoulos, Cultes et rites de passage en Macédoine (MEAETHMATA, 19), Athènes, 1994.

${ }^{23}$ Cf. N.G.L. Hammond, op. cit. à la n. 9, p. 424-41.

24 Cf. M.B. Hatzopoulos et Louisa D. Loukopoulou, Recherches sur les marches orientales des Téménides, 1ère partie (ME 1 ETHMATA, 11), Athènes, 1992, p. 15-25. 
qui n'était pas inclus dans son enceinte, sinon par des rapports tels avec les maîtres de l'arrière-pays qu'ils ne faisaient pas craindre aux Méthonéens un blocus terrestre mettant en péril leurs communications avec la mer, comme cela s'est produit pendant la Guerre du Péloponnèse ${ }^{25}$ ? Quant à Pydna, il s'agissait probablement d'une ville dès son origine macédonienne, ainsi que l'absence de tradition concernant sa métropole pouvait faire soupçonner ${ }^{26}$, et que les trouvailles de l'époque archaïque tendent maintenant à confirmer ${ }^{27}$.

L'attitude positive du royaume macédonien face aux entreprises coloniales et à l'immigration à la fin de la période archaïque et au début de la période classique s'est vérifiée à plusieurs reprises. Ainsi Amyntas Ier proposa-t-il au Pisistratide Hippias, alors exilé d'Athènes, de venir s'installer en Anthémonte ${ }^{28}$. Il s'agissait probablement d'un projet d'occupation commune de cette fertile contrée $^{29}$. Au siècle suivant, les Mycéniens et les Histiéens, victimes respectivement des Argiens et des Athéniens, furent invités à venir faire de la Macédoine leur nouvelle partie ${ }^{30}$. Mais déjà l'orientation de la politique macédonienne était en train de changer : si l'installation des colons venus de la Grèce du Sud à l'intérieur du royaume était plus que jamais souhaitée, la fondation de nouvelles colonies dans ses abords par les grandes métropoles était regardée de plus en plus avec méfiance et hostilité. C'est qu'entre temps la Macédoine avait fait les frais de l'expansionnisme athénien et tout particulièrement dans ses marches orientales, qui à la suite de la retraite perse, s'étaient avancées des bords de l'Axios à ceux du Strymon. La prise d'Ennéa Hodoi par Alexandre Ier en 478 fut suivie deux ans plus tard par celle d'Eion par Cimon. Athènes bloquait ainsi la sortie de la vallée du Strymon. En 465, dix mille hommes envoyés d'Athènes entreprenaient la colonisation de cette vallée que les Macédoniens avaient dû abandonner. Cette entreprise ambitieuse, malgré de graves revers, fut finalement couronnée par la fondation d'Amphipolis, à Ennéa Hodoi en 436. Entre temps toutes les colonies des Grecs du Sud à l'Est du fond du Golfe Thermaïque étaient progressivement annexées à l'empire athénien. Les encouragements prodigués par Athènes à tous les ennemis, internes et externes, du roi macédonien, Perdiccas II, ne pouvaient pas ne pas lui faire craindre que les jours de l'indépendance de son pays ne soient comptés ${ }^{31}$.

La débâcle perse avait marqué un tournant décisif aussi pour les Chalcidiens

${ }^{25}$ M.B. Hatzopoulos, D. Knoepfler et Véra Marigo-Papadopoulos, Deux sites pour Méthone de Macédoine, dans BCH, 114, 1990, p. 639-68.

${ }^{26}$ Cf. U. Kahrstedt, Städte in Makedonien, dans Hermes, 81, 1953, p. 85-88 ; J.N. Kalléris, op. cit. à la n. 15 , p. 594

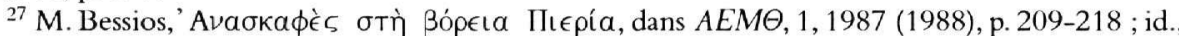

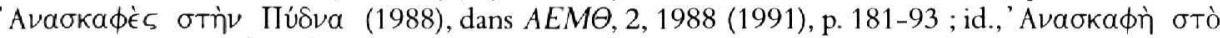

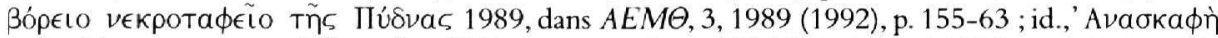

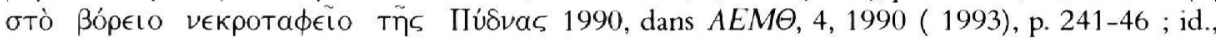

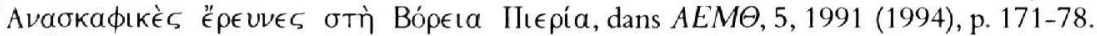

${ }^{28}$ Hérod., 5, 94, 1.

${ }^{29}$ M.B. Hatzopoulos - L.D. Loukopoulou, op. cit. à la n. 24, p. 23.

30 Paus., 7, 25, 6 et Théopompe, FGrHist., 115 F 387.

31 Pour tout ce qui précède, cf. N.G.L. Hammond dans N.G.L. Hammond et G.T. Griffith, $A$ History of Macedonia, vol. II, Oxford, 1974, p. 98-104 et 115-23. 
de Thrace. Les Perses en retraite leur livrèrent en 479 la ville bottiéenne d'Olynthe, inaugurant ainsi l'expansion chalcidienne vers l'arrière-pays. De même, très vite, il devint évident que l'expansionnisme athénien sur le littoral septentrional de la Mer Egée ne menaçait pas seulement le royaume arriéré de Macédoine, mais autant - sinon plus - les poleis coloniales qu'avaient fondées d'autres métropoles de la Grèce du Sud, telles Chalcis ou Corinthe. La réaction de défense immédiate fut le synœcisme d'Olynthe et, sinon immédiatement, du moins peu après, la création de la Ligue Chalcidienne, et la conséquence à peine différée l'ouverture du premier véritable front de la Guerre du Péloponnèse. Les faits sont connus. Sous l'impulsion de Perdiccas II, en 432, les Chalcidiens abandonnèrent leurs petites agglomérations maritimes en faveur d'Olynthe, qui devint ainsi le siège de leurs institutions communes. Le roi macédonien, qui en quelque sorte tint le nouvel état sur les fonts baptismaux, lui céda - ne fût-ce qu'à titre précaire - ses possessions en Mygdonie orientale, près du lac Bolbè, que de toute façon il avait probablement du mal à soustraire à l'emprise athénienne. (Aréthuse, Bormiscos, Kallipolis ${ }^{32}$ ?)

Sans relater les péripéties de la Guerre du Péloponnèse en Grèce du Nord, il convient de rappeler que la parade imaginée par Perdiccas ne fit pas sentir immédiatement ses effets bénéfiques. Bien au contraire, le roi dut voir les armées athéniennes sillonner son royaume, assiéger Pydna, attaquer Béroia, capturer Therma et annexer durablement à leur empire non seulement Méthone, ancienne colonie érétrienne, mais aussi Hérakleion, ville de son propre royaume, sans parler des menaces également graves que firent peser sur le royaume argéade les alliés locaux d'Athènes : Illyriens, Lyncestes, Élimiotes, Thraces, voire les propres frère et neveu de Perdiccas ${ }^{33}$. Cependant, sur son lit de mort, bien avant le désastre sicilien et la capitulation d'Athènes, le roi macédonien aurait pu estimer que son action avait été positive. L'empire athénien en Grèce du Nord n'était qu'un souvenir dont la nouvelle Ligue Chalcidienne, qu'il avait contribué à créer, empêcherait à tout jamais la reconstitution. Le verrou d'Amphipolis, qui bloquait l'expansion macédonienne vers l'Est, avait sauté. La nouvelle Amphipolis indépendante avait toutes les raisons de s'appuyer sur la Macédoine pour repousser d'éventuelles tentatives athéniennes de la ramener sous son contrôle et l'on peut parier que le roi prit soin de rendre inefficace sa participation à la campagne du général athénien Euétion contre cette cité en 414 av. J.C. ${ }^{34}$

Perdiccas ne se rendait probablement pas compte qu'Athènes avait seméiles graines d'une ivraie que la création de la Ligue Chalcidienne, loin d'éradiquer, faisait croître et embellir, voire que son propre enfant spirituel s'avèrerait une menace bien plus redoutable et immédiate que la lointaine Athènes.

Le premier symptôme, apparemment isolé, fut la révolte et la sécession de sa bonne ville de Pydna en 410, que le roi Archélaos, son successeur, dut ramener

\footnotetext{
${ }^{32}$ M. Zahrnt, op. cit. à la n. 12, p. 49-57.

${ }^{33}$ Cf. N.G.L. Hammond, op. cit. à la n. 31, p. 122-36.

${ }^{34}$ Thuc., 7, 9 .
} 
au giron du royaume après un long siège et au prix de sa transplantation vers l'intérieur des terres ${ }^{35}$.

Moins de vingt ans plus tard, le roi macédonien Amyntas III devait conclure un traité par lequel il cédait aux Chalcidiens - en principe temporairement - ses possessions d'Anthémonte et de Mygdonie orientale et leur concédait d'humiliants privilèges commerciaux ${ }^{36}$. Quant une dizaine d'années plus tard il demanda la restitution de ses états, non seulement il essuya un refus catégorique, mais il vit les cités de son propre royaume, les une après les autres, jusques et y compris Pella, embrasser la cause chalcidienne et, au nom de la liberté, faire sécession $^{37}$. Il est vrai qu'en 379 Amyntas avait récupéré la majeure partie de ses anciennes possessions ; Olynthe capitulait devant les Spartiates et leurs alliés locaux et la Ligue Chalcidienne était dissoute ${ }^{38}$. Mais ce ne fut qu'un bref répit.

En 370 la Ligue s'était reconstituée et le mouvement autonomiste était redevenu assez fort, pour que le prétendant Pausanias pût avec l'aide des Chalcidiens rallier sans difficulté les cités d'Anthémonte et de Mygdonie. Iphicrate sauva momentanément la situation, mais deux ans plus tard une coterie d'aristocrates macédoniens originaires des villes côtières assassina le roi légitime Alexandre II, pour le remplacer par le régent Ptolémée d'Alôros, qui était acquis à leur cause. Cela explique pourquoi, quand Perdiccas III put se débarrasser de l'usurpateur en 365 , il changea en même temps d'orientation diplomatique et, s'alliant à la Confédération athénienne reconstituée, fit la guerre aux Chalcidiens et à leurs alliés amphipolitains, qui depuis trente ans avaient représenté la menace principale pour l'intégrité territoriale du royaume. Mais encore une fois le remède s'avéra pire que le mal. Les Athéniens annexèrent Pydna et Méthone et Perdiccas, par un retournement spectaculaire des alliances, se mua en protecteur d'Amphipolis, où il dépêcha une garnison ${ }^{39}$.

Philippe II, arrivé au pouvoir après la mort de son frère, sembla amorcer un virage diplomatique en évacuant Amphipolis et en faisant la paix avec Athènes, voire en faisant miroiter à l'ancienne métropole l'engagement de lui livrer la cité rebelle contre Pydna. La menace d'une alliance attico-macédonienne décida les Chalcidiens à donner à Philippe carte blanche au sujet d'Amphipolis en échange de Potidée et d'Anthémonte. Les Athéniens, médusés, virent Philippe non seulement prendre d'assaut Amphipolis mais bientôt après Pydna (357), suivie trois ans plus tard par Méthone ${ }^{40}$. La Ligue Chalcidienne, alliée inquiète de la Macédoine, restait désormais l'unique représentant du fait colonial dans la région et la seule rivale du royaume argéade. Pas pour longtemps. Philippe, non sans avoir été provoqué, déclara la guerre à la Ligue et en deux ans (349-48)

35 Cf. U. Kahrstedt, art. cit. à la n. 26, p. 89-91 et M.B. Hatzopoulos, La Béotie et la Macédoine à l'époque de l'hégémonie thébaine : le point de vue macédonien, dans La Béotie antique, Paris, 1985, p. 253.

${ }^{36}$ Syll $^{3} 135$.

${ }^{37}$ Xen., Hell., 5, 2, 13 ; cf. M.B. Hatzopoulos, art. cit. à la n. 35, p. 248.

${ }^{38}$ M. Zahrnt, op. cit. à la n. 12, p. 91-97.

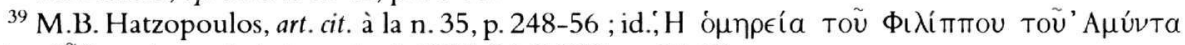
otǐs $\theta \tilde{\eta} ß \in \varsigma$, dans Archaiognosia, 4, 1985-86 [1989], p. 37-58.

${ }^{40}$ M.B. Hatzopoulos, op. cit. à la n. 19, p. 64-67. 
conquit tous ses territoires et rasa sa capitale Olynthe ${ }^{41}$. Et Toynbee de rappeler qu'il n'y avait pas eu un tel acte de barbarie dans l'histoire hellénique depuis la destruction de Sybaris ${ }^{42}$.

L'acte - certes barbare mais moins isolé dans l'histoire grecque que ne le veut l'historien britannique ${ }^{43}$ - de Philippe ne se comprend qu'à la lumière des rapports du royaume macédonien avec les cités coloniales de la Grèce du Nord, voire avec le phénomène urbain dans ces régions. Les rois macédoniens étaient dès le départ pris dans un dilemme sans solution. S'ils voulaient être capables de résister à leurs voisins agressifs, grecs ou barbares, et de maintenir ne fût-ce qu'un semblant de suzeraineté sur les royaumes "alliés " de la Haute Macédoine, ils devaient mobiliser les ressources naturelles et humaines de leur pays, vastes mais inexploitées. À cette époque-là, tout comme aujourd'hui, la "modernisation " était le prix qu'il fallait payer pour survivre. Mais la modernisation au Ve ou au IVe siècle a.C. signifiait avant tout l'introduction massive de méthodes, institutions et attitudes développés dans les États les plus avancés de cette époque, les cités de la Grèce du Sud, qui étaient indissolublement liées au phénomène de la polis dans tous ses aspects : économique, social, politique, militaire et culturel. Les rois téménides eussent sans doute préféré laisser de côté l'aspect politique de la modernisation, mais ce n'était pas plus possible à cette époque qu'aux temps modernes. Inévitablement, l'introduction massive d'institutions et attitudes économiques, militaires et culturelles façonnées dans le monde des poleis dans un État unitaire fondamentalement " ethnique " ne pouvait qu'avoir - et eut effectivement - des conséquences perturbatrices dans le domaine politique. L'idéal de la polis classique était fait d'indépendance, voire d'autarcie, et son monde clos, qui exigeait une fidélité et une dévotion totales, ne laissait pas de place pour une allégeance à des entités externes comme l'ethnos ou le roi ${ }^{44}$.

On ne saurait trop insister sur l'étroitesse des rapports et sur les destins parallèles des États téménide et chalcidien. Fondée sur une initiative téménide et en partie sur territoire téménide, la Ligue Chalcidienne devint le partenaire naturel et en même temps la rivale dangereuse des Macédoniens. On ne peut s'empêcher de penser que ce projet qui réussit au-delà des espoirs les plus extravagants de ses initiateurs, finit par représenter d'abord un idéal malheureusement inaccessible de modernité hellénique pour les rois téménides et plus tard un pôle d'attraction irrésistible pour leurs sujets les plus " progressistes ». Alors que les rois téménides peinaient pour encourager le développement urbain avec le minimum inévitable d'autonomie qu'il impliquait, sans briser l'unité de l'ethnos macédonien et sans saper les fondements de leur autorité, les cités à peine viables qu'ils avaient contribué à unir, tendaient à devenir de plus en plus un véritable ethnos, sans perdre aucun des avantages de la modernité hellénique qu'elles avaient hérité de leur origine civique : une citoyenneté commune, le droit de

${ }^{41}$ M. Zahrnt, op. cit. à la n. 12, p. 104-111; cf. M.B. Hatzopoulos, op. cit. à la n. 12, p. 76-77.

${ }^{42}$ A. Toynbee, op. cit. à la n. 4, p. 486.

${ }^{43}$ Cf. G.T. Griffith dans N.G.L. Hammond et G.T. Griffith, A History of Macedonia, II, Oxford, 1979 , p. 325.

${ }^{44}$ Cet aspect de la question est developpé dans la monographie signalée à la n. 21 . 
mariage et de propriété sur tout le territoire de la Ligue, des revenus communs, des droits de douane fédéraux, un système commun de taxation locale, une monnaie commune, un calendrier commun, une armée commune, des magistratures communes (la meilleure illustration en est une série de prêtres fédéraux connus par les actes de vente trouvés un peu partout sur le territoire de la Ligue, de Spartolos à Stolos et d'Arnai (?) à Toronè), mais en même temps une vie civique très active au niveau local, chaque cité retenant sa personnalité juridique propre, l'essentiel de ses lois et de ses magistrats avec à leur tête l'épistate ${ }^{45}$. Il n'est point étonnant que les rois téménides se sentissent à la fois jaloux d'un équilibre aussi enviable et menacés par lui. Tant que la Ligue Chalcidienne exerçait cette attraction irrésistible sur les cités du royaume, Philippe ne pouvait se sentir en sécurité. C'est le sens du message qu'il aurait envoyé aux Olynthiens, quand il se trouvait à peine à huit kilomètres de leur ville : " de deux choses l'une, soit eux ne devraient plus habiter à Olynthe, soit lui plus jamais en

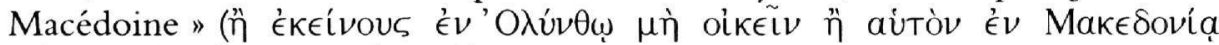

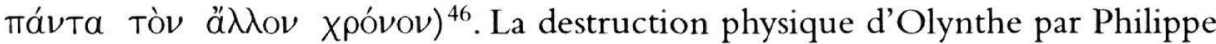
constitue peut-être la reconnaissance la plus manifeste de la fascination mortellement dangereuse que la Ligue Chalcidienne exerçait sur les souverains et le peuple macédoniens.

Toynbee tient pour acquis que rien ne subsista de la Ligue Chalcidienne. "Cette fois ", écrit-il, « les Chalcidiens furent traités sans pitié. Leurs terres furent confisquées et eux-mêmes furent soit vendus comme esclaves soit déportés dans l'arrière-pays. Ainsi la confédération qui, une génération auparavant, avait aspiré à devenir la nouvelle grande puissance du Nord fut-elle complètement anéantie ${ }^{47} \%$. Ces lignes écrites il y presque soixante ans correspondent-elles vraiment à la réalité historique que révèlent les découvertes des six dernières décennies et décrivent-elles fidèlement la politique du royaume macédonien envers les colonies des Grecs du Sud dans la région?

Un acte de donation royale de terres de l'ancienne Ligue Chalcidienne ${ }^{48}$ et une série d'actes de vente d'Amphipolis ${ }^{49}$ m'ont donné récemment l'occasion de me pencher sur le sort des colonies du littoral septentrional de la Mer Egée conquises par Philippe II. J'ai constaté que l'ancienne colonie athénienne avait conservé la grande majorité de ses habitants, son statut de cité, voire ses anciennes magistratures (épistate, polémarques etc.), mais qu'elle avait dû recevoir un contingent important de colons macédoniens. adopter le calendrier macédonien, en un mot s'intégrer au royaume et devenir une cité macédonienne à part entière, avec les mêmes droits et les mêmes devoirs que n'importe quelle cité du Vieux Royaume à l'Ouest de l'Axios ${ }^{50}$. Quant aux cités de la

${ }^{45}$ Sur l'épistate, voir M.B. Hatzopoulos, op. cit. à la n. 12, p. 62, 68 .

${ }^{46}$ Dém., 9, 11.

${ }^{47}$ A. Toynbee, op. cit. à la n. 4, p. 486.

${ }^{48}$ M.B. Hatzopoulos, Une donation du roi Lysimaque (MEAETHMATA, 5), Athènes, 1988, p. 17 18.

${ }^{49}$ M.B. Hatzopoulos, op. cit. à la n. 19, p. 14-54.

${ }^{50}$ M.B. Hatzopoulos, op. cit. à la n. 19, p. 74-82. 
Chalcidique, leur sort fut moins cruel que ne le prétend Démosthène ${ }^{51}$ ou ne le pense Toynbee. Tout d'abord, les cités de la Chalcidique orientale et en particulier de l'Aktè, qui avaient réussi à se tenir en dehors de l'État chalcidien, conservèrent leur autonomie et semblent, dans un premier temps du moins, être devenues des "alliés ", en fait, bien sûr, des vassaux du roi macédonien. Parmi les membres de la Ligue, seules Olynthe et Stagire furent physiquement détruites et leurs populations vendues ou déportées. Les autres furent seulement conquises et asservies. Il y eut certainement des confiscations étendues et probablement toutes les cités de la Ligue cessèrent d'exister en tant qu'entités "politiques ". Leurs anciens citoyens, sans doute sans cadre institutionnel bien défini, restèrent sur place, coexistant tant bien que mal avec les Macédoniens qui avaient reçu des domaines prélevés sur les terres confisquées et converties en terres royales ${ }^{52}$. Mais même Stagire fut relevée de ses ruines et rétablie dans la plénitude de ses droits ${ }^{53}$ et Cassandreia, la nouvelle fondation de Cassandre, fut l'héritière de la Ligue Chalcidienne, car elle fut le résultat du synœecisme de la plupart des anciennes cités de la Ligue qui avaient survécu en tant que centres urbains et des anciens citoyens d'Olynthe survivants et de leurs descendants ${ }^{54}$.

Il est vrai que l'historien britannique pourrait répondre que plus que la survivance physique, c'est la permanence culturelle et institutionnelle des colonies du Nord de l'Egée qu'il avait en vue. Cependant, il faut souligner que non seulement Amphipolis, mais aussi finalement Cassandreia huit décennies plus tard, ainsi que les cités du Nord et de l'Est de la Chalcidique, c'est-à-dire pratiquement tout le domaine colonial grec de la région, furent incorporées dans le royaume macédonien. N'est-il pas légitime de se poser la question de savoir si cette incorporation eut pour résultat l'élimination pure et simple - du moins sur le plan culturel et institutionnel, sinon physique - des vaincus au profit des vainqueurs ou s'il en est résulté une synthèse originale, mêlant les traditions des deux puissances helléniques de la région?

Dans le domaine culturel je m'arrêterai à l'élément le plus fondamental et le plus tangible, celui de la langue. Déjà bien avant le règne de Philippe II, dans toute la région thraco-macédonienne les alphabets épichoriques avaient cédé leur place à l'alphabet ionien oriental, qui depuis la fin du Ve siècle avait fini par être adopté même à Athènes ${ }^{55}$. En revanche, à première vue du moins, la polymorphie dialectale se perpétuait jusqu'au règne de Philippe II. La version chalcidienne, découverte à Olynthe, du traité de Philippe II avec le Koinon conclu en 357 , est rédigée dans le dialecte ionien eubéen local ${ }^{56}$. De même, le décret d'Amphipolis condamnant à l'exil perpétuel les adversaires politiques du roi macédonien et datant de la même année, est rédigé dans le dialecte ionien de la

\footnotetext{
51 Dém., 9, 26.

52 M.B. Hatzopoulos, op. cit. à la n. 48, p. 43-45.

53 cf. M.B. Hatzopoulos, op. cit. à la n. 48 , p. 45.

${ }^{54}$ M.B. Hatzopoulos, op. cit. à la n. 48, p. 45-47.

55 Cf. A. Panayotou, Ancient Greek Dialects and Scripts in Northern Greece, dans les actes du First International Conference on Greek Linguistics (à paraître).

${ }^{56}$ Syll. ${ }^{3} 135$.
} 
région strymonienne ${ }^{57}$. D'un autre côté les dédicaces de la reine Eurydice, épouse d'Amyntas III et mère de Philippe II, à Eukleia semblent avoir été rédigées dans un dialecte du Nord-Ouest ${ }^{58}$, de mêrne que les légendes monétaires du père (Amyntas III) ou du frère (Perdiccas III) de Philippe ${ }^{59}$, ou encore les defixiones du IVe siècle découvertes récemment à Pella et ailleurs ${ }^{60}$.

À peine quelques années après le début du règne de Philippe II, tous les documents, tant officiels que privés découverts dans le royaume désormais élargi sont rédigés dans la koinè attique. On attribue souvent cette réforme à Philippe et on l'explique par l'influence directe d'Athènes sur la Macédoine ${ }^{61}$. En fait c'est à l'influence d'Amphipolis et de la Ligue Chalcidienne vaincues et annexées qu'il faudra attribuer l'origine de cette réforme. En réalité, l'ionien des textes officiels, traités, décrets, légendes monétaires, n'est qu'une vitrine, une langue d'apparat, qui cache mal les réalités linguistiques de la vie quotidienne. À Amphipolis et à Argilos les actes de vente contemporains, voire antérieurs aux textes officiels, sont rédigés entièrement en koinè attique, seule la déclinaison des noms propres conservant des traits ioniens ${ }^{62}$. En Chalcidique, si la plupart des actes de vente préservent une apparence dialectale, elle est entièrement due à la morphologie nominale, qui, si elle était mise à part, laisserait peu d'éléments dialectaux dans ces textes ${ }^{63}$. Qui plus est, il y a au moins un acte de vente d'Olynthe entièrement rédigé en koinè attique ${ }^{64}$ et même des textes officiels, tel le traité entre Amyntas III et les Chalcidiens, trahissent des habitudes linguistiques relevant non pas du dialecte local mais de la koine ${ }^{65}$. La preuve de l'origine coloniale et non pas directement athénienne de la koinè adoptée par la chancellerie macédonienne est maintenant fournie par le document officiel le plus ancien du règne de Philippe II, le bornage mygdonien datant des années 357-350, dont le vocalisme trahit des habitudes linguistiques locales ${ }^{66}$. Dans le domaine linguistique du moins, la Grèce coloniale du littoral septentrional de la Mer Égée se perpétua à travers la grande Macédoine de Philippe II, l'empire d'Alexandre et les États des Diadoques jusqu'au grec de nos jours.

57 Syll. ${ }^{3}$ 194, avec nos remarques (op. cit. à la n. 19, p. 77-78).

58 Bull. Epigr., 1984, 249 ; 1987, 654 ; 1988, 828 ; 1991, 386 ; cf. M.13. Hatzopoulos, op. cit. à la n. 19, p. 79, n. 4.

${ }^{59}$ Cf. C. Brixhe et A. Panayotou, L'atticisation de la Macédoine : L'une des sources de la koiné, dans Verbum, 10, 1988, p. 251.

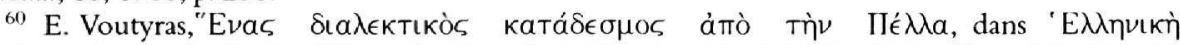
$\Delta \iota a \lambda \epsilon \kappa т о \lambda о \gamma i ́ a, 3,1992-1993$, p. 43-48 ; cf. S. Moschonisioti, A.Ph. Christidès et Th. Glaraki,

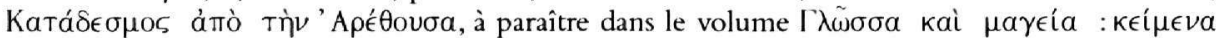

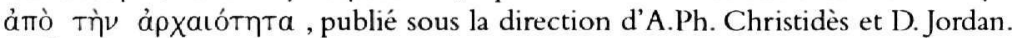

${ }^{61}$ Cf. J.N. Kalléris, op. cit. à la n. 15, p. 503-531.

62 M.B. Hatzopoulos, op cit. à la n. 19 , p. 77-80.

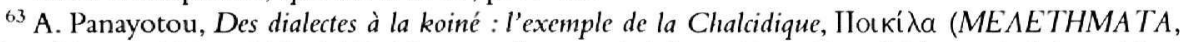
10), Athènes, 1990 , p. 225 ; M.B. Hatzopoulos, op. cit. à la n. 19, p. 80 ; cf. id., op. cit. à la n. 12, p. 40-50.

${ }^{64}$ D.M. Robinson, Inscriptions from Macedonia, 1938, dans TAPA, 69, 1938, p. 52-54, $\mathrm{n}^{\circ} 6$.

${ }^{65}$ Syll. $^{3} 135$; cf. M.B. Hatzopoulos, op. cit. à la n. 12 , p. 48-49.

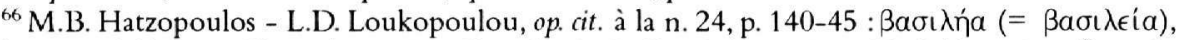

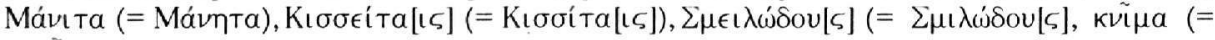

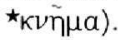


Se pourrait-il que dans le domaine institutionnel, les belles réussites de cette Grèce coloniale n'aient pas été non plus purement et simplement anéanties ? Dans les Nouveaux Territoires au-delà de l'Axios l'élément le plus important était constitué par les anciennes colonies ioniennes qui, au Ve siècle, avaient fait partie de l'Empire athénien et qui, par la suite, avaient fait partie de la Ligue Chalcidienne. Philippe II eut le génie de comprendre qu'il n'était ni possible ni souhaitable de réprimer l'aspiration à l'autonomie civique, qui était inscrite dans l'héritage politique des anciennes colonies et qui avait gagné aussi les cités proprement macédoniennes du royaume, mais qu'un gouvernement central assez fort et sûr de lui-même pourrait intégrer, domestiquer le mouvement civique qui s'était développé jusqu'alors en opposition aux autorités centrales, et l'utiliser à ses propres fins.

En affaires intérieures, le roi était certainement plus conservateur que les descriptions dramatisées d'un Théopompe ne le laisseraient entendre ${ }^{67}$. Il favorisa sans doute l'urbanisation et n'hésita pas à avoir recours à des transferts massifs de population afin d'atteindre ses buts ${ }^{68}$. Néanmoins, il était en général respectueux des traditions locales et il fit le meilleur usage - et le plus étendu - des structures existantes. La Haute Macédoine tout comme l'arrière-pays crestonien, bisalte ou édonien à l'Est de l'Axios continua d'être un pays de villages. Cependant, les ethnè, c'est-à dire les unités territoriales de la Haute Macédoine, furent assimilées à des poleis et les communautés indigènes à l'Est de l'Axios furent regroupées en sympolities assimilées, elles aussi, à des cités ${ }^{69}$. Aux cités grecques, anciennes ou nouvelles, trop périphériques pour être incorporées dans la communauté macédonienne, comme par exemple Philippes, il accorda un statut d' "allié " et les laissa s'occuper de leurs propres affaires ${ }^{70}$. Quant aux autres cités grecques d'origine coloniale, en dehors d'un petit nombre qui avaient été détruites et qui devaient rester en ruine pendant une génération ou deux ${ }^{71}$, il les intégra dans la communauté macédonienne, parfois les refondant entièrement, parfois, quoique ni nécessairement ni toujours, en y introduisant des colons provenant du Vieux Royaume ${ }^{72}$.

Ainsi entre les ethnè de la Haute Macédoine et les sympolities indigènes assimilées à des cités, les anciennes cités coloniales et les cités proprement macédoniennes du Vieux Royaume, la Macédoine - si l'on excepte les terres royales fut dans son intégralité subdivisée en cités et territoires civiques. Il y eut une politeia macédonienne commune, comme il y avait eu une politeia chalcidienne commune, mais les politeiai des cités et des unités territoriales de la Haute

\footnotetext{
${ }^{67}$ FGrHist., 115, F 224 et 225.

${ }^{68}$ Cf. J.R. Ellis, Population Transplants by Philip II, dans Makedonika, 9, 1969, p. 9-17.

${ }^{69}$ Cf. M.13. Hatzopoulos, Épigraphie et villages en Grèce du Nord : ethnos, polis et kome en Macédoine, dans L'epigrafia del villaggio, Faenza, 1993, p. 151-71.

${ }^{70}$ Cf. M.B. Hatzopoulos, Décret pour un bienfaiteur de la cité de Philippes, dans BCH, 117, 1993, p.

${ }^{71}$ En particulier Olynthe et Stagire ; cf. M.B. Hatzopoulos, op. cit. à la n. 48, p. 44-45.

${ }^{72}$ Le cas le mieux connu est celui d'Amphipolis ; cf. M.B. Hatzopoulos, op. cit. à la n. 19, p. 80-
} 321-24. 86. 
Macédoine continuèrent à subsister ${ }^{73}$. L'existence d'une législation commune à la Macédoine toute entière n'empêchait pas chaque cité de continuer à voter ses propres lois ${ }^{74}$. Il y eut cependant un calendrier commun à l'ensemble du pays, comme jadis il y avait eu un calendrier chalcidien commun. S'il n'y eut pas un prêtre éponyme fédéral, comme dans la Ligue Chalcidienne, Philippe imposa le prêtre d'Asclépios local comme le prêtre éponyme dans toutes les cités du royau$\mathrm{me}^{75}$. On peut se demander où le roi avait puisé l'inspiration de cette réforme grandiose qui fit de la Macédoine un pays de cités. Un puissant indice montre qu'une de ses sources d'inspiration, sinon sa source principale, fut la défunte Ligue Chalcidienne. Le titre du magistrat suprême - et parfois unique - dans chaque cité, qui devint à tel point partie intégrante de l'héritage macédonien qu'il apparut comme une caractéristique distinctive des fondations macédoniennes d'Asie, fut celui de l'épistate ${ }^{76}$. Or tel avait été aussi le titre du magistrat suprême dans les anciennes cités coloniales, membres de la Ligue Chalcidienne ou associées à elle, que nous connaissons par les actes de vente de Stôlos, d'Argilos ou d'Amphipolis ${ }^{77}$.

Pourrais-je, enfin, émettre une hypothèse ? Depuis la parution de l'oeuvre monumentale de Georges Le Rider sur le monnayage d'or et d'argent de Philippe II, on date la frappe des premières monnaies d'argent de ce roi et par conséquent aussi la réforme monétaire par laquelle il fit adopter l'étalon " thraco-macédonien " qui avait cours notamment dans la Ligue Chalcidienne et à Amphipolis, du tout début de son accession au pouvoir, traditionnellement située en 359 , mais en fait en $360^{78}$. A la lumière, cependant, de la récente publication d'un bronze de Philippe montrant qu'au tout début de son règne ce dernier avait continué les types, et probablement aussi l'étalon de Perdiccas III $^{79}$, ne pourrait-on pas se demander si sa réforme et le début de son monnayage d'argent ne dateraient pas de deux ou trois ans plus tard et s'il ne faudrait pas les mettre en rapport avec la prise d'Amphipolis en 357 ? Ce ne serait qu'un autre exemple de la survie insoupçonnée du monde colonial grec des côtes septen-

${ }^{73}$ Non seulement les Macédoniens sont désignés par l'ethnique de leur cité (ou de leur ethnos de la Haute Macédoine), mais aussi un Macédonien d'une cité s’installant dans une autre a le sta-

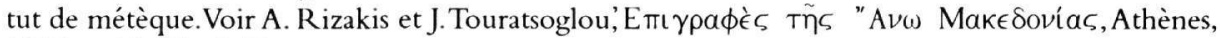
1985 , p. $85-87, \mathrm{n}^{\circ} 87$.

${ }^{74}$ Voir en particulier Ph. Gauthier et M.B. Hatzopoulos, La loi gymnasiarchique de Béroia (MEAETHMATA, 16), Athènes 1983.

${ }^{75}$ Cf. M.B. Hatzopoulos et Louisa D. Loukopoulou, Morrylos : cité de la Crestonie (ME $\Lambda E T H^{-}$ MATA, 7), Athènes 1988, p. 65 ; id., op. cit. à la n. 24, p. 112-13 ; Bull. Epigr., 1988, 847.

${ }^{76}$ En attendant la parution de la monographie mentionnée à la note 21, voir M.B. Hatzopoulos, op. cit. à la n. 19, p. 74-76 et 80-81; cf. Sylvie Le Bohec, Les épistates des rois Antigonides, dans Ktema, 11,1986 , p. 281-88.

${ }_{77}^{77}$ M.B. Hatzopoulos, op. cit. à la n. 12 , p. 30 et 68 ; id., op. cit. à la n. 48, p. 74-76.

${ }^{78}$ M.B. Hatzopoulos, The Oleveni Inscription and the Dates of Philip II's Reign, dans Philip II, Alexander the Great and the Macedonian Heritage, Washington D.C., 1982, p. 21-42, et maintenant, id., La lettre royale d'Olévéni, dans Chiron, 35,1985.

${ }^{79}$ C.A. Hersh, An Unpublished Coin of Philip II of Macedonia, from his First Issue of Bronzes, dans AJN, Second Series, 1, 1989, p. 33-36, pl. 3. 
trionales de la Mer Egée à travers la Macédoine nouvelle de Philippe, qui plus que le triomphe pur et simple d'un régime "barbare et réactionnaire ${ }^{80}$ » représentait une synthèse originale des "Argéades et des Chalcidiens d'Eubée ${ }^{81}$ ", des deux éléments qui transformèrent l'ancien pays des dieux et des barbares en Grèce du Nord.

${ }^{80}$ A. Toynbee, op. cit. à la n. 4, p. 489.

${ }^{81}$ Strab.,VII, frg. 11. 